\title{
Indicadores de obesidade em jovens com Síndrome de Down
}

\author{
Indicators of obesity in young people with Down Syndrome
}

\author{
Fábia Freire, ${ }^{1 *}$ Leonardo Trevisan Costa, ${ }^{2}$ José Irineu Gorla ${ }^{3}$
}

ARTIGO ORIGINAL | ORIGINAL ARTICLE

\begin{abstract}
O objetivo do presente estudo foi comparar indicadores de crescimento e obesidade em função do sexo, como também, verificar a correlação das variáveis de dois indicadores de obesidade, índice de massa corporal (IMC) e percentual de gordura $(\% \mathrm{G})$, em crianças e adolescentes com síndrome de Down. A amostra foi constituída de 104 crianças e adolescentes matriculados em instituições especializadas do Estado de São Paulo, sendo 58 meninos e 46 meninas com idades entre 7 e 17 anos. Para a obtenção do IMC e \%G foram utilizadas as medidas antropométricas de massa corporal, estatura e pregas cutâneas subescapular e tricipital. Os resultados foram analisados pelo coeficiente de correlação de Spearman e apresentaram valores de rho $=0.87$ entre os escores brutos de IMC e \%G sendo encontrada forte correlação entre as variáveis. Assim, conclui-se que o IMC em relação ao \%G demonstrou ser um bom indicador de sobrepeso e obesidade para pessoas com síndrome de Down.

Palavras-chave: obesidade, IMC, pregas cutâneas, síndrome de Down
\end{abstract}

ABSTRACT

The aim of this study was to compare growth and obesity parameters by gender, as well as check the correlation on the variables of two obesity parameters, body mass index (BMI) and \% body fat in children and adolescents with Down syndrome. The sample was composed by 104 children and adolescents, 58 boys and 46 girls ranging between 07 and 17 years-old, all students enrolled in specialized institutions of the State of São Paulo. BMI and \% body fat were calculated after collecting a few anthropometric measures, such as body mass, height and skinfolds (subscapular and triceps). The results were analyzed by rank correlation coefficient Spearman. Correlations between the raw scores of BMI and $\%$ body fat was 0.87 , i.e., a strong effect. Thus, results demonstrated that BMI is strongly associated to \% body fat, being a good procedure to assess overweight and obesity in people with Down syndrome.

Keywords: obesity, BMI, skinfold, Down syndrome

Artigo recebido a 29.10.2012; $1^{\text {a }}$ Revisão 12.04.2013; 2 ${ }^{\text {a }}$ Revisão 08.11.2013; Aceite 11.11.2013

${ }^{1}$ Universidade Paranaense - UNIPAR, Toledo - PR, Brasil

${ }^{2}$ Centro Universitário de Votuporanga, UNIFEV, Votuporanga - SP, Brasil

${ }^{3}$ Departamento de Estudos de Atividade Física Adaptada da Universidade Estadual de Campinas, UNICAMP, Campinas, SP, Brasil

* Autor correspondente: Universidade Paranaense, Rua Santos Dumont, 2171 - Centro, Toledo - PR, CEP: 85903-170, Brasil; E-mail: fabiafreire@uol.com.br 


\section{INTRODUÇÃO}

Os estudos em pessoas com Síndrome de Down (SD) vêm crescendo e despertando interesse dos pesquisadores nos últimos tempos, tendo em vista que é a anomalia cromossómica mais comum entre os neonatos e que a expectativa de sobrevida tem aumentado (Martin, Mendes, \& Hessel, 2011; Roizen \& Patterson, 2003).

De acordo com Pueschel (1990) a maioria das pessoas com SD apresenta anormalidades determinadas por fatores genéticos, étnicos e nutricionais, tais como patologias no coração, hipotonia muscular e défice na hormona tiroide. A prevalência de sobrepeso e obesidade é maior nesta população comparada à população em geral sem síndrome (Silva, Santos, \& Martins, 2006).

Estudos (Cronk et al., 1998; Eichstaedt \& Lavay, 1992; Styles, Cole, Dennis, \& Preece, 2002) têm demonstrado que a prevalência de sobrepeso e obesidade sofre incremento com o avanço da idade em pessoas com SD, tornandose fundamental a prevenção da obesidade nessa população para diminuir os riscos de comorbidades.

Mesmo em jovens sem síndrome, o sobrepeso e a obesidade nas últimas décadas têm aumentado substancialmente e se constituído em fator de preocupação na área da saúde pública (Guedes \& Guedes, 2003; Williams et al., 1992). Diversos são os fatores que contribuem para este cenário, dentre eles, o excesso de massa corporal e de gordura corporal em jovens, que aumentam o risco de se tornarem adultos com sobrepeso ou obesidade. Nesta perspetiva, o desenvolvimento de fatores de risco que podem predispô-los a distúrbios metabólicos e funcionais tende a uma maior incidência (Guedes \& Guedes, 2003).

Eichstaedt e Lavay (1992) destacaram a tendência da obesidade em crianças e adultos com deficiência intelectual (DI) ser mais eleva$\mathrm{da}$, especialmente em crianças e adolescentes com deficiência intelectual leve e com SD, aumentando o risco de enfarte agudo do miocárdio, hipertensão arterial e diabetes, relacio- nando a uma expectativa de vida mais baixa.

A expectativa de vida das pessoas com esta síndrome, que em 1929, era de 9 anos, nos anos 90 aumentou para acima de 50 anos, sendo que o envelhecimento precoce nesta população parece acelerado e o óbito ocorre entre a $5^{\mathrm{a}}$ e $6^{\mathrm{a}}$ década de vida (Sherril, 1998). Esta diminuição da mortalidade pode ser explicada pelo avanço na medicina e maior conhecimento a respeito desta população, porém com muitas lacunas a serem estudadas e preenchidas (Allt \& Howell, 2003).

Especificamente na população com SD, existem poucos estudos quanto à composição corporal em crianças e adolescentes. Na maioria dos estudos, ênfase é dada ao desenvolvimento de tabelas referenciais de curvas de crescimento físico para crianças e adolescentes com SD (Cronk et al., 1998; Myrelid, Gustafsson, Ollars, \& Annerén, 2002; Styles et al., 2002) e ao índice de massa corporal (IMC) em adultos com SD (Fujiura, Fitzsimons, Mark, \& Chicoine, 1997; Melville, Cooper, McGrother, Thorp, \& Collacott, 2005; Rubin, Rimmer, Chicoine, Braddock, \& Mcguire, 1998).

O IMC é um índice antropométrico muito utilizado por pesquisadores que buscam compreender melhor as dimensões corporais do ser humano. A Organização Mundial da Saúde (OMS, 2000) adota o IMC como um preditor confiável de sobrepeso e obesidade na população em geral. Muito embora o IMC possa não identificar os diferentes componentes da composição corporal, é um método utilizado como indicador de obesidade devido ao seu baixo custo (Januário, Nascimento, \& Barazetti, 2008). Entretanto, o IMC varia substancialmente de acordo com a idade cronológica, etnia e sexo, além de apresentar menor exatidão para diagnosticar sobrepeso e obesidade em crianças e adolescentes (Loveday, Thompson \& Mitchell, 2012; Wang \& Wang, 2002).

De acordo com Mendonça e Pereira (2008), no passado, o elevado índice de prevalência de obesidade e sobrepeso em pessoas com SD era atribuído ao uso confuso das classificações de IMC. Contudo, como esses indivíduos apresen- 
tam composição corporal diferente da população sem a síndrome, não poderiam ser utilizados os mesmos valores convencionais de classificação de estado nutricional (Martin et al., 2011).

González-Agüero, Vicente-Rodríguez, Ara, Moreno e Casajús (2011), avaliaram a precisão de equações para predizer a percentagem de gordura $(\% \mathrm{G})$ através de pregas cutâneas comparado com a pletismografia em crianças e adolescentes com SD. Para tanto, a amostra foi constituída por 12 meninas e 16 meninos espanhóis com idades entre 10 e 20 anos. As equações analisadas foram obtidas a partir dos protocolos de Durnin e Rahaman (1967), Durnin e Womersley (1974), Jhonston et al. (1988), Brook (1971) e Slaughter et al. (1988). Os autores observaram que somente as equações propostas por Slaughter et al. (1988) não apresentaram diferença significativa na predição de \%G da amostra quando comparado com a pletismografia $(p=0.583)$, além de ser a única equação analisada que não demonstrava heterocedasticidade $(p=0.596)$.

Desta forma, dentro do contexto da necessidade de discussão de equações e a escassez de estudos no contexto brasileiro que abordem esta temática, o objetivo deste estudo foi comparar indicadores de crescimento e obesidade em função do sexo, como também, verificar a correlação das variáveis de dois indicadores de obesidade, índice de massa corporal (IMC) e percentual de gordura $(\% \mathrm{G})$, em crianças e adolescentes com SD. Acredita-se que o conjunto destas informações possa fornecer indicações preciosas sobre a necessidade de futuras investigações e oferecer uma perspetiva de análise complementar do estado nutricional de indivíduos com SD.

\section{Participantes}

\section{MÉTODO}

Trata-se de um estudo descritivo e transversal. A amostra investigada foi constituída por 104 crianças e adolescentes com síndrome de Down (SD) selecionadas por conveniência, de ambos os sexos, com idades entre 7 e 17 anos
(11.18 \pm 2.64 anos), avaliados em instituições especializadas do município do Estado de São Paulo, sendo 58 do sexo masculino. A coleta de dados ocorreu entre os meses de agosto e novembro de 2010, nas instituições onde os mesmos encontravam-se matriculados.

Foram incluídos na pesquisa os indivíduos com SD regularmente matriculados nas instituições, cujos pais ou responsáveis deram anuência através da assinatura do termo de Consentimento Livre e Esclarecido, de acordo com a resolução n. 196/96 do Conselho Nacional de Saúde. Não participaram do estudo os sujeitos que se recusaram por qualquer motivo a realizar a avaliação, bem como aqueles que estavam ausentes no dia da coleta de dados.

A pesquisa foi aprovada pelo Comitê de Ética e Pesquisa da UNICAMP (protocolo n. ${ }^{\circ}$ 492/2010).

\section{Instrumentos e Procedimentos}

As variáveis antropométricas utilizadas no estudo para obtenção do IMC foram massa corporal e a estatura, medidas através de uma balança digital (Wiso $\mathrm{W} 801^{\circledR}$ ), com precisão de $100 \mathrm{~g}$ e capacidade de medida de $180 \mathrm{~kg}$ e um estadiómetro compacto com precisão de $0.1 \mathrm{~cm}$ $\left(\mathrm{Wiso}^{\circledR}\right)$, respetivamente. Todos os indivíduos foram avaliados descalços e com o mínimo de vestimenta possível. Posteriormente, o IMC foi determinado pela equação IMC = Massa corporal $(\mathrm{kg}) /$ Estatura $\left(\mathrm{m}^{2}\right)$.

A composição corporal foi obtida através da técnica de espessura das pregas cutâneas Tricipital (TR) e Subescapular (SE); a avaliação foi efetuada em triplicado e realizada no lado direito do corpo, pelo mesmo avaliador, sendo considerado o valor médio. Para tal, foi utilizado o compasso Harpenden ${ }^{\circledR}$ (Harpenden Instruments, Marsden, UK) de acordo com as recomendações de Slaughter et al. (1984). A gordura corporal foi estimada pelas equações de Slaughter et al. (1988) de acordo com o somatório das pregas cutâneas $\left(\sum \mathrm{DC}\right) \mathrm{TR}$ e SE, a seguir:

Para o $\sum \mathrm{DC} \geq 35 \mathrm{~mm}$ (todas as idades): 
Meninos: $\% \mathrm{G}=0.783 \times\left(\sum \mathrm{DC}\right)+1.6$

Meninas: $\% \mathrm{G}=0.546 \times\left(\sum \mathrm{DC}\right)+9.7$

Para o $\sum \mathrm{DC}<35 \mathrm{~mm}$ :

Meninos (Pré-púbere):

$\% \mathrm{G}=1.21\left(\sum \mathrm{DC}\right)-0.008\left(\sum \mathrm{DC}\right)^{2}-1.7$

Meninos (Púbere):

$\% \mathrm{G}=1.21\left(\sum \mathrm{DC}\right)-0.008\left(\sum \mathrm{DC}\right)^{2}-3.4$

Meninos (Pós-púbere):

$\% \mathrm{G}=1.21\left(\sum \mathrm{DC}\right)-0.008\left(\sum \mathrm{DC}\right)^{2}-5.5$

Meninas (qualquer nível maturacional):

$\% \mathrm{G}=1.33\left(\sum \mathrm{DC}\right)-0.013\left(\sum \mathrm{DC}\right)^{2}-2.5$

\section{Critérios de Referência}

O critério de referência do IMC utilizado foi o recomendado por Cole, Belizzi, Flegal e Dietz (2000), que determina pontos de cortes específicos para crianças e adolescentes de acordo com sexo e idade cronológica, construído a partir de dados de diferentes países, inclusive o Brasil.

Em relação ao percentual de gordura, optou-se pelos valores sugeridos por Williams et al. (1992), o qual indica condições de magreza, eutrofia, sobrepeso e obesidade (> $25 \%$ para meninos e $>30 \%$ para as meninas) e que tem sido utilizado amplamente em estudos nacionais com crianças e adolescentes (Brito, Silva Júnior, Coelho, \& França, 2012; Buonani et al., 2011; Fernandes et al., 2007; Veira, Alvarez, Marins, Sichieri, \& Veiga, 2006).

\section{Análise Estatística}

O tratamento estatístico dos dados foi realizado através do pacote computadorizado $\mathrm{R}$ Commander 1.6-3 (Rcmdr) no software R plus, versão 2.12.1. Inicialmente, utilizou-se a estatística descritiva (mediana e amplitude interquartil), uma vez que a maioria das variáveis (idade, massa corporal, pregas cutâneas e \%G) não foi considerada normal a partir do teste de normalidade de Shapiro-Wilk. Para identificar diferenças entre os sexos, foi aplicado o teste de Mann-Whitney para dados não paramétricos e $t$ de Student para dados paramétricos.

Posteriormente, foi utilizado o índice de coeficiente de correlação de Spearman (rho) para avaliar a correlação entre a classificação do sobrepeso e obesidade segundo os escores de IMC e \%G, e os pontos de cortes sugeridos por Cole et al. (2000) e Williams et al. (1992). O nível de significância estatística adotado foi de $p \leq 0.05$.

\section{RESULTADOS}

A Tabela 1 sumariza as características descritivas da amostra estudada apresentando os valores de mediana da idade, massa corporal, estatura, IMC, pregas cutâneas tricipital e subescapular e percentual de gordura com seus respetivos intervalos interquartil. A tabela apresenta também os resultados do teste Mann Whitney referente à comparação entre crianças e adolescentes do sexo masculino e feminino quanto a essas variáveis.

No presente estudo observou-se que, em ambos os sexos, a classificação de obesidade com base no IMC foi inferior àquela obtida pelas pregas cutâneas, ou seja, $18.97 \%$ dos meninos e $36.96 \%$ das meninas foram classificados como obesos pelo IMC. Já em relação às pregas cutâneas, os valores indicaram $39.66 \%$ dos meninos e $54.35 \%$ das meninas acima dos valores indicados para gordura corporal $(>25 \%$ para os meninos e $>30 \%$ para as meninas).

Em relação ao coeficiente de correlação de postos de Spearman quando analisado os valores entre os pontos de cortes para classificação de sobrepeso e obesidade sugeridos por Cole et al. (2000) e Williams et al. (1992) foi encontrada correlação de rho $=0.87 \quad(p<0.05)$, demonstrando forte correlação entre as duas variáveis, IMC e \%G.

\section{DISCUSSÃO}

O presente estudo correlacionou indicadores de obesidade, avaliado pelo IMC e \%G, em jovens com síndrome de Down de ambos os sexos, tendo também comparado estes indicadores de crescimento e obesidade em crianças e adolescentes com SD.

Embora a Organização Mundial da Saúde (OMS, 2000) recomende a utilização do IMC para diagnosticar gordura corporal alguns pes- 
Tabela 1

Características da amostra em valores de mediana e amplitude interquartil segundo medidas aferidas por sexo

\begin{tabular}{cccc}
\hline Variáveis & Masculino $(\mathrm{n}=58)$ & Feminino $(\mathrm{n}=46)$ & $p$ \\
\hline Idade (anos) & $12.0(9.2-14.0)$ & $10.0(8.0-13.0)$ & 0.06 \\
Massa Corporal $(\mathrm{kg})^{\mathrm{a}}$ & $38.8(29.1-50.7)$ & $35.2(26.4-49.8)$ & 0.36 \\
Estatura $(\mathrm{cm})^{\mathrm{b}}$ & $131.5(124.0-46.8)$ & $126.9(117.2-136.0)$ & $0.01^{*}$ \\
IMC $\left(\mathrm{kg} / \mathrm{m}^{2}\right)^{\mathrm{b}}$ & $21.8(18.9-24.6)$ & $22.3(18.4-28.5)$ & 0.17 \\
Tricipital $(\mathrm{mm})^{\mathrm{a}}$ & $11.7(9.2-17.1)$ & $17.1(12.4-22.2)$ & $0.01^{*}$ \\
Subescapular $(\mathrm{mm})^{\mathrm{a}}$ & $12.4(8.7-20.7)$ & $20.4(9.6-30.4)$ & $0.03^{*}$ \\
Somatório $(\mathrm{mm})^{\mathrm{a}}$ & $23.9(17.8-37.7)$ & $38.1(21.5-51.7)$ & $0.01^{*}$ \\
\%G $^{\mathrm{a}}$ & $22.0(16.5-30.0)$ & $30.5(20.1-37.9)$ & $0.01^{*}$ \\
\hline
\end{tabular}

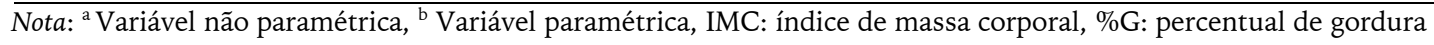

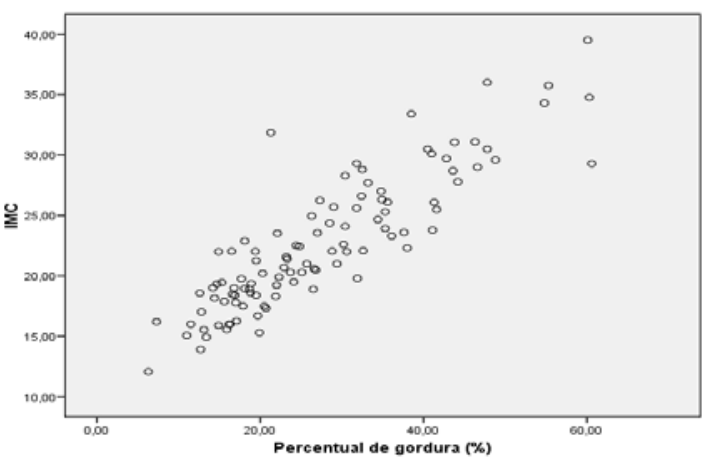

Figura 1. Coeficiente de correlação de Spearman (rho) entre os valores brutos de IMC e \%G

quisadores têm questionado o uso deste índice (Bergman et al., 2011; Ricardo \& Araújo, 2002). Desse modo, deve-se ter cautela ao utilizá-lo como indicativo único de diagnóstico de sobrepeso e obesidade, uma vez que, existem dúvidas quanto à sua capacidade de diagnosticar o que realmente se propõe em populações sem síndrome (Ricardo \& Araújo, 2002), e em especial, em um público específico com características fenotípicas diferenciadas como a SD.

Foi observado no presente estudo que o IMC considerou dentro dos padrões estabelecidos para obesidade $18.97 \%$ dos meninos e $36.96 \%$ das meninas e em relação às pregas cutâneas, os valores indicaram $39.66 \%$ dos meninos e $54.35 \%$ das meninas acima dos valores indicados para gordura corporal.
Os resultados de um estudo realizado com a população típica, que analisou o IMC e \%G em escolares entre 8 e 10 anos, demonstraram que o IMC considerou como normal $15 \%$ das meninas e $21 \%$ dos meninos pelos critérios estabelecidos para obesidade, enquanto o \%G considerou-os como obesos. O índice Kappa foi de 0.43 e 0.50 , respetivamente, para meninos e meninas apresentando concordância moderada (Januário et al., 2008).

Glaner (2005) investigou o IMC e pregas cutâneas como indicativo da gordura corporal numa amostra de 694 meninas e 716 meninos sem síndrome entre 11 e 18 anos de idade. Os achados indicaram que somente $48.98 \%$ das meninas e $57.32 \%$ dos meninos foram classificados simultaneamente pelo IMC e pregas cutâneas, cujo índice Kappa indicou uma concordância fraca entre as três categorias de classificação da gordura corporal, sendo que as medidas de pregas cutâneas indicaram $46.12 \%$ das meninas e $28.87 \%$ dos meninos acima do recomendado em relação à gordura corporal e o IMC classificou-os dentro do padrão adequado.

Vale ressaltar que estudos sobre a composição corporal em pessoas com SD ainda são pouco pesquisados, sendo o público adulto, geralmente, o foco principal destes estudos. Outra questão que deve ser enfatizada é a falta de protocolos específicos para a população jovem com SD. 
Pesquisa realizada por Sardinha, Going, Teixeira e Lohman (1999) mencionou que a ausência de critérios apropriados para identificação de sobrepeso e obesidade desde a infância e adolescência na população típica têm contribuído para o aumento da prevalência da obesidade na idade adulta.

O que vem reforçar esta preocupação é demonstrado no estudo de Rubin et al. (1998) que verificaram segundo critérios estabelecidos no National Health and Nutrition Examination Survey e Healthy People 2000, que $45 \%$ dos homens e $56 \%$ das mulheres adultos com SD estavam acima do peso, o que demonstra números substancialmente superiores aos encontrados na população geral brasileira que é de $12.5 \%$ dos homens e $16.9 \%$ das mulheres, segundo o Instituto Brasileiro de Geografia e Estatística (IBGE, 2010). Observando que o Brasil possui hoje cerca de 190 milhões de habitantes, destes, mais de 45 milhões declararam ter algum tipo de deficiência e mais de dois milhões e meio com deficiência intelectual. Tais informações devem ser observadas por profissionais da área da saúde e pessoas que trabalham com pessoas com SD, sobretudo quando se pretende adotar medidas para diminuição dos níveis de gordura corporal, sobrepeso e obesidade, características frequentemente comuns a esta população e tão claramente associadas à doenças que prejudicam a saúde e qualidade de vida.

No estudo de Myrelid et al. (2002) cujo objetivo era a elaboração de gráficos de crescimento para crianças e adolescentes de 0 a 18 anos com SD, foram apresentados gráficos de crescimento de massa corporal e os resultados apontaram que os meninos tiveram massa corporal média ao nascer de $3.0 \mathrm{~kg}$, correspondente a -1.2 desvio-padrão (DP). A massa corporal média aos 18 anos de idade foi de $61 \mathrm{~kg}$, correspondente a -0.4 DP de acordo com o padrão sueco. O IMC acima de $25 \mathrm{~kg} / \mathrm{m}^{2}$ foi observado em $31 \%$ dos meninos e $36 \%$ das meninas até os 18 anos de idade, demonstrando uma tendência ao sobrepeso e a obesidade.
Em nosso estudo $30.43 \%$ das meninas e $34.48 \%$ dos meninos apresentaram sobrepeso.

No tocante a estudos específicos sobre a espessura de pregas cutâneas relacionada ao \%G não foi encontrada na literatura nacional algo semelhante para pessoas com SD. Conforme os achados do presente estudo, o IMC quando comparado ao $\% \mathrm{G}$ aferido pelo método de espessura de pregas cutâneas, apresentou forte correlação para meninos e meninas com SD, respetivamente, da faixa etária de 7 a 17 anos. Oliveira et al. (2006) corroboram com estes achados ao avaliar a precisão dos pontos de corte propostos por Cole et al. (2000) comparado com o \%G, estimado pelo método de absortometria radiológica de dupla energia (DXA), em 418 adolescentes sem deficiência de ambos os sexos. Os autores verificaram que os critérios estabelecidos a partir do IMC são eficientes na identificação de adolescentes obesos e eutróficos.

Em um estudo realizado com adolescentes e adultos jovens com SD, Bandini, Fleming, Scampini, Gleason e Must (2012) analisaram a validade da utilização do IMC para identificar o excesso de adiposidade corporal para esta população. Os pontos de cortes analisados de IMC foram os propostos pela National Center for Disease Control and Prevention (CDC) e foram comparados com os valores de \%G mensurados pelo DXA. Os autores relatam que o ponto de corte para obesidade (percentil 95) apresentou sensibilidade e especificidade de $71 \%$ e $96 \%$ respetivamente. O valor de predição positiva foi de $83 \%$ e negativa $92 \%$, com eficiência de $91 \%$. Em relação aos pontos de corte para sobrepeso (percentil 85), foi encontrada sensibilidade e especificidade de $100 \%$ e $60 \%$ respetivamente. O valor de predição positiva foi de $41 \%$ e negativa $100 \%$, com eficiência de $69 \%$. Com isso, os autores concluem que o IMC se apresenta como uma boa ferramenta para identificar o excesso de gordura corporal em adolescentes e adultos jovens com SD, sendo que, o protocolo da CDC é mais preciso para identificar obesidade do que sobrepeso. 
Quando analisada a diferença entre meninos e meninas, foi observada diferença estatística de $\% \mathrm{G}$, sendo que o sexo feminino atingiu valores maiores de mediana quando comparado ao sexo masculino. Esses achados estão de acordo com o estudo realizado por GonzálezAgüero, Ara, Moreno, Vicente-Rodríguez, e Casajús (2011) que investigaram as diferenças de massa gorda e magra entre meninos e meninas com SD. Para tanto, os autores avaliaram 31 crianças e adolescentes com SD de 10 a 19 anos de idade. Foi observado que o grupo do sexo feminino apresentou maiores valores $(p<0.05)$ de massa gorda mensurada por meio do DXA corporal total, tronco, membros superiores e inferiores.

Algumas limitações deste estudo estão relacionadas com as próprias características da SD, como por exemplo, o comprometimento intelectual que dificulta o entendimento das informações em algumas situações bem como a dificuldade da coleta de dados, principalmente em relação às pregas cutâneas, como também, a escassez de estudos nesta área.

Apesar das considerações realizadas, o estudo apresenta relevância para a área da educação física adaptada e da saúde, uma vez que seus achados permitem um maior entendimento sobre as limitações de métodos utilizados na população em geral quando empregados em indivíduos com SD.

\section{CONCLUSÕES}

A partir dos resultados encontrados pode-se concluir que o IMC apresenta forte correlação com o \%G, demonstrando ser um bom indicador de sobrepeso e obesidade para pessoas com SD.

Entretanto, sugerem-se novas investigações a fim de corroborar e complementar os dados aqui apresentados, de forma a determinar e confirmar pontos de cortes associados a problemas de saúde em crianças e adolescentes com SD, que possam contribuir para a promoção de saúde e qualidade de vida desta população.
Agradecimentos:

Nada a declarar.

\section{Conflito de Interesses:}

Nada a declarar.

\section{Financiamento:}

Auxílio através de bolsa de Mestrado da Coordenação de Aperfeiçoamento de Pessoal de Nível Superior (CAPES), Brasil.

\section{REFERÊNCIAS}

Allt, J. E., \& Howell, C. J. (2003). Down's syndrome. British Journal of Anaesthesia, 3(3), 83-86. doi: 10.1093/bjacepd/mkg083

Bandini, L. G., Fleming, R. K., Scampini, R., Gleason, J., \& Must, A. (2012). Is body mass index a useful measure of excess body fatness in adolescents and young adults with Down syndrome? Journal of Intellectual Disability Research, 57, 1050-1057. doi:10.1111/j.13652788.2012.01605.x

Bergman, R. N., Stefanovski, D., Buchanan, A. T., Summer, A. E., Reynolds, J. C., Sebring, N. G., ... Watanabe, R. M. (2011). A better index of body adiposity. Obesity, 19(5), 1083-1089. doi: 10.1038/oby.2011.38

Brito, A. K. A., Silva Júnior, F.L., Coelho, L.S., \& França, N.M. (2012). Nível de atividade física e correlação de massa e percentual de gordura em adolescentes escolares da cidade de Teresina- PI. Revista Brasileira de Atividade Física e Saúde, 17(3), 212-216.

Brook, C. G. D. (1971). Determination of body composition of children from skinfold measurements. Archives of Disease Childhood, 46, 182184.

Buonani, C., Fernandes, R. A., Bueno, D. R., Bastos, K. N., Segatto, A. F. M., Silveira, L. S., \& Freitas Júnior, I. F. (2011). Desempenho de diferentes equações antropométricas na predição de gordura corporal excessiva em crianças e adolescentes. Revista de Nutrição, 24(1), 41-50. doi: 10.1590/S1415-52732011000100004

Cole, T. J., Bellizzi, M. C., Flegal, K. M, \& Dietz, W. H. (2000). Establishing a standard definition for child overweight and obesity worldwide: In- 
ternational survey. British Medical Journal, London, 320, 1240-1247. doi: 10.1136/bmj.320. 7244.1240

Cronk, C., Crocker, A. C., Pueschel, S. M., Shea, A. M., Zackai, E., Pickens, G., \& Reed, R. B. (1998). Growth charts for children with Down syndrome: 1 month to 18 years age. Pediatrics, $81(1), 102-110$.

Durnin, J. V., \& Rahaman, M. M. (1967). The assessment of the amount of fat in the human body from measurements of skinfold thickness. British Journal of Nutrition, 21 (3), 681-689.

Durnin, J. V., \& Womersley, J. (1974). Body fat assessed from total body density and its estimation from skinfold thickness: Measurements on 481 men and women aged from 16 to 72 years. British Journal of Nutrition, 32(1), 77-97.

Eichstaedt, C. B., \& Lavay, B. W. (1992). Physical activities for individuals with mental retardation - Infancy through adulthood. Champaign, Illinois: Human Kinetics.

Fernandes, R. A., Rosa, C. S. C., Silva, C. B., Bueno, D. R., Oliveira, A. R., \& Freitas Júnior, I. F. (2007). Desempenho de diferentes valores críticos de índice de massa corporal na identificação de excesso de gordura corporal e obesidade abdominal em adolescentes. Revista da Associação Médica Brasileira, 53(6), 515-519. doi: 10.1590/S0104-42302007000600019

Fujiura, G., Fitzsimons, N., Mark, B., \& Chicoine, B. (1997). Predictors of BMI among adults with Down syndrome: The social context of health promotion. Research in Development Disabilities, 18, 261-274. doi: 10.1016/S0891-4222(97) 00008-5

Glaner, M. F. (2005). Índice de massa corporal como indicativo da gordura corporal comparado às dobras cutâneas. Revista Brasileira de Medicina do Esporte, 11(4), 243-246. doi: 10.1590/S151786922005000400008

González-Agüero, A., Ara, I., Moreno, L. A., Vicente-Rodríguez, G., \& Casajús, J. A. (2011). Fat and lean masses in youths with Down syndrome: Gender differences. Research in Development Disabilities, 32, 1685-1693. doi: 10.1016/ j.ridd.2011.02.023

González-Agüero, A., Vicente-Rodríguez, G., Ara, I., Moreno, L. A., \& Casajús, J. A. (2011). Accuracy of prediction equations to assess percentage of body fat in children and adolescents with Down syndrome compared to air displacement plethysmography. Research in Development Disa- bilities, 32(5), 1764-1769. doi: 10.1016/j.ridd. 2011. 03.006

Guedes, D. P., \& Guedes, J. E. R. P. (2003). Controle do peso corporal: Composição corporal, atividade física e nutrição ( $2^{\mathrm{a}}$ ed.). Rio de Janeiro: Shape.

Instituto Brasileiro de Geografia e Estatística (2010). Censo demográfico 2010: Resultados gerais da amostra. Retirado de http://www. ibge.gov.br/home/presidencia/noticias/impren sa/ppts/0000000847310412201231572748398 5.pdf

Januário, R. S. B., Nascimento, M. A., \& Barazetti, L. K. (2008). Índice de massa corporal e dobras cutâneas como indicadores de obesidade em escolares de 8 a 10 anos. Revista Brasileira de Cineantropometria \& Desempenho Humano, 10(3), 266-270.

Johnston, J. L., Leong, M. S., Checkland, E. G., Zuberbahler, P. C., Conger, P. R., \& Quinney, H. A. (1988). Body fat assessed from body density and estimated from skinfold thickness in normal children and children with cystic fibrosis. American Journal of Clinical Nutrition, 48, 1362-1366.

Loveday, S. J., Thompson, J. M. D., \& Mitchell, E. A. (2012). Bioelectrical impedance for measuring percentage body fat in young persons with Down syndrome: Validation with dual-energy absorptiometry. Acta Pcediatrica, 101, e491e495. doi: 10.1111/j.1651-2227.2012.02821.x

Martin, J. E. S., Mendes, R. T., \& Hessel, G. (2011). Peso, estatura e comprimento em crianças e adolescentes com Síndrome de Down: Análise comparativa de indicadores antropométricos de obesidade. Revista de Nutrição, 24(3), 485-492. doi: 10.1590/S1415-52732011000300011

Melville, C. A., Cooper, S. A., McGrother, C. W., Thorp, C. F., \& Collacott, R. (2005). Obesity in adults with Down syndrome: A case-control study. Journal Intellectual Disability Research, 49(2), 125-133. doi: 10.1111/j.1365-2788.20 04.00616.x

Mendonça, G. V., \& Pereira, F. D. (2008). Medidas de composição corporal em adultos portadores de síndrome de Down. Revista Brasileira de Educação Física e Esporte, 22(3), 201-210.

Myrelid, A. Gustafsson, J., Ollars, B., \& Annerén, G. (2002). Growth charts for Down's Syndrome from birth to 18 years of age. Archives of Disease in Childhood, 87, 97-103.

Oliveira, F. L., Taddei, J. A., Escrivão, M. A., Cobayashi, F., Barros, M. E., Vítolo, M. R., ... Anco- 
na-López, F. (2006). Accuracy of obesity diagnosis in Brazilian adolescents: Comparison of Cole et al and Must et al criteria with DXA percentage of fat mass. Nutrición Hospitalaria, 21 (4), 484-490.

Organização Mundial da Saúde (2000). World Health Organization consultation on obesity. Obesity: Preventing and Managing the Global Epidemic. (Technical Report Series 894). Genève: Autor.

Pueschel, S. M. (1990). Clinical aspects of down syndrome from infancy to adulthood. American Journal of Medical Genetics, 7, 52-56. doi: 10.1002/ajmg. 1320370708

R Development Core Team. (2010). R: A language and environment for statistical computing. Vienna: $\mathrm{R}$ Foundation for Statistical Computing.

Ricardo, D. R., \& Araújo, C. G. S. (2002). Índice de massa corporal: Um questionamento científico baseado em evidências. Arquivos Brasileiros de Cardiologia, 79(1), 61-69.

Roizen, N. J., \& Patterson, D. (2003). Down's Syndrome. Lancet, 361(9365), 1281-1289. doi: 10.1016/S0140-6736(03) 12987-X

Rubin, S. S., Rimmer, J. H., Chicoine, B., Braddock, D., \& Mcguire, D. (1998). Overweight prevalence in persons with Down Syndrome. Mental Retardation, 36, 175-181. doi: 10.1352/00476765(1998)036<0175:OPIPWD>2.0.CO;2

Sardinha, L. B., Going, S. B.,Teixeira, P. J., \& Lohman, T. G. (1999). Receiver operating characteristic analysis skinfold thickness and arm girth for obesity screening in children and adolescents. American Journal of Clinical Nutrition, 70, 1090-1095.

Sherrill, C. (1988). Adapted physical activity, recreation and sport: Crossdisciplinary and lifespan ( $\left.5^{\mathrm{a}} \mathrm{ed}.\right)$. Boston, Mass: WCB/Mc Graw-Hill.

Silva, D. L., Santos, J. A. R., \& Martins, C. F. (2006). Avaliação da composição corporal em adultos com síndrome de Down. Arquivos de Medicina, 20(4), 103-110.

Slaughter, M. H., Lohman, T. G., Boileau, R. A., Horswill, C. A., Stillman, R. J., Van Loan, M., \& Bemben, D. A. (1988). Skinfold equations for estimation of body fatness in children and youth. Human Biology, 60 (5), 709-723.

Slaughter, M. H., Lohman, T. G., Boileau, R. A., Stillman, R. J., Van Loan, M., Horswill, C. A., \& Wilmore, J. H. (1984). Influence of maturation on relationship of skinfolds to body density: A cross-sectional study. Human Biology, 56(4), 681-689.

Styles, M. E., Cole, T. J., Dennis, J., \& Preece, M. A. (2002). New cross sectional stature, weight, and head circumference references for Down's syndrome in the UK and Republic of Ireland. Archives of Disease in Childhood, 87, 104-108. doi: 10.1136/adc.87.2.104

Vieira, A. C. R., Alvarez, M. M., Marins, V. M. R., Sichieri, R., \& Veiga, G.V. (2006). Desempenho de pontos de corte do índice de massa corporal de diferentes referências na predição de gordura corporal em adolescentes. Cadernos de Saúde Pública, 22(8), 1681-1690. doi: 10.1590/ S0102-311X2006000800016

Wang, Y., \& Wang, J. Q. (2002). A comparison of international references for the assessment of child and adolescent overweight and obesity in different populations. European Journal of Clinical Nutrition, 56, 973-982.

Williams, D. P., Going, S. B., Lohman, T. G., Harsha, D. W., Srinivasan, S. R., Webber, L., \& Berenson, G. S. (1992). Body fatness and risk for elevated blood pressure, total cholesterol, and serum lipoprotein ratios in children and adolescents. American Journal of Public Health, 82(3), 358-363. doi: 10.2105/AJPH.82.3.358

Todo o conteúdo da revista Motricidade está licenciado sob a Creative Commons, exceto quando especificado em contrário e nos conteúdos retirados de outras fontes bibliográficas. 